\title{
Inventario de gases con efecto invernadero de la Universidad Técnica Nacional sede Atenas, Costa Rica y su relación con el número de estudiantes, período 2012-2015
}

\author{
Rodney Orlando Cordero S. \\ Decano de la Universidad Técnica Nacional. rcordero@utn.ac.cr
}

Recibido: 00 noviembre 2018

\section{RESUMEN}

Inventory of gases with greenhouse effect of the Universidad Técnica Nacional in Atenas, Costas Rica and its relationship with the number of students, period 2012-2015

Se cuantificaron las principales fuentes emisoras de $\mathrm{CO}_{2} \mathrm{e}$ en la Universidad Técnica Nacional sede Atenas (UTNSA) y se relacionó con el promedio por estudiantes desde el 2012 y hasta 2015 como línea base para planificar el balance de carbono en busca de la neutralidad. Se utilizó la metodología del Grupo Intergubernamental de Expertos en Cambio Climático (IPCC) planteada en el 2006 y los factores de emisión publicados por el Instituto Meteorológico Nacional (IMN) 2016. Se identificaron ocho fuentes de emisiones, seis directas y dos indirectas, siendo estas: semovientes, residuos ordinarios, combustibles, aguas residuales, fertilizantes nitrogenados, electricidad, gas licuado de petróleo (GLP) y extintores de $\mathrm{CO}_{2}$, según su orden de importancia. Las tres primeras fuentes emitieron el 93\% del total de emisiones de la UTN-SA. Al desglosar las emisiones se determinan tres patrones: las que aumentan las emisiones, las que se mantienen y las que disminuyen a pesar del incremento de estudiantes. En general las emisiones totales incrementan al aumentar el número de estudiantes, pero al mismo tiempo las emisiones por estudiante disminuyen.

Palabras claves: Huella de carbono, neutralidad, indicadores ambientales, $\mathrm{tCO}_{2}$ e, efecto invernadero, inventario.

\footnotetext{
ABSTRACT

The main sources of $\mathrm{CO}_{2}$ e emissions were quantified in the National Technical University, Athens campus (UTN-SA) and it was related to the average per student from 2012 to 2015 as a baseline to accomplish the carbon neutrality
}

Aceptado: 00 febrero 2018

national policy. The methodology used was the one recommended by the Intergovernmental Panel on Climate Change (IPCC) 2006, and emission factors published by the National Meteorological Institute (IMN) 2016. Eight emission sources were identified, six direct and two indirect: livestock, ordinary waste, fuel, sewage water, nitrogen fertilizers, electricity, liquefied petroleum gas (LPG), and $\mathrm{CO}_{2}$ fire extinguishers, in order of importance. The first three sources issued $93 \%$ of the total emissions at the UTNSA. Three patterns are determined to break down emissions: which increase emissions, which are kept and which decrease despite the increase of students. In general the total emissions increase by increasing the number of students, but at the same time emissions per student decreases.

Key words: trace of carbon neutrality, environmental indicators, $\mathrm{tCO}_{2} \mathrm{e}$, greenhouse, inventory.

\section{INTRODUCCIÓN}

Una de las principales fuentes de contaminación es la generada por los llamados gases con efecto invernadero (GEI), los cuales, al liberarse a la atmósfera, crean una capa que limita la liberación de calor de la superficie de la tierra, lo que a su vez aumenta la temperatura del planeta, a este efecto se le conoce como calentamiento global (IPCC, 2007). Seis son los principales gases que favorecen el efecto invernadero en la atmósfera: dióxido de carbono $\left(\mathrm{CO}_{2}\right)$, monóxido de carbono $(\mathrm{CO})$, metano $\left(\mathrm{CH}_{4}\right)$, óxido nitroso $\left(\mathrm{N}_{2} \mathrm{O}\right)$, óxidos de nitrógeno $\left(\mathrm{N}_{\mathrm{x}} \mathrm{O}\right)$ y componentes orgánicos volátiles diferentes al metano, como hidrofluorocarbonos (HFC), perfluorocarbonos (PFC) y hexafluoruro de azufre $\left(\mathrm{SF}_{6}\right)$ (Kioto, 
1997). Según el Grupo Intergubernamental de Expertos sobre el Cambio Climático, foro establecido en el marco de la Naciones Unidas en 1988, la temperatura del planeta ha aumentado $0,74^{\circ} \mathrm{C}$ de 1906 a 2004 y se espera que aumente entre $1,4^{\circ} \mathrm{C}$ y $5,8^{\circ} \mathrm{C}$ en el presente siglo (IPCC, 2007). No obstante, al 2016 el incremento registrado llego a $1,1^{\circ} \mathrm{C}$, lo que sitúa el aumento global de las temperaturas a una distancia peligrosamente próxima al "techo" máximo de 1,5 grados fijado por el Acuerdo de París (Fresneda, 2017). El gobierno de Costa Rica propuso que para el 2021 el país sería carbono neutral, lo cual implica que las emisiones de carbono al ambiente serán iguales a la capacidad de fijación del país (Dobles, 2008). Este compromiso, impulsó a empresas, organizaciones, instituciones y universidades, incluida la Universidad Técnica Nacional sede Atenas (UTN-SA) a estimar su huella de carbono y proponer posibles medidas de mitigación. Como punto de comparación, el promedio per cápita nacional es de $2,41 \mathrm{tCO}_{2} \mathrm{e}$ (IMN, 2012) y para el 2030, se proyecta bajar a $1,7 \mathrm{tCO}_{2}$ e mediante la implementación de acciones de disminución y fijación de gases efecto invernadero (MINAE, 2016). Para desarrollar este estudio se planteó una hipótesis de trabajo: 1) a mayor número de estudiantes hay menor generación $\mathrm{CO}_{2}$ e por año por estudiante.

\section{Materiales y Métodos}

Área de estudio. La Universidad Técnica Nacional sede Atenas (UTN-SA) se ubica en la proyección 9॰55'35.76” - 9॰56’33.5” " N y 84²3'35.09" - 84²1'42.88” W, específicamente en el caserío de Balsa, cantón de Atenas en la provincia de Alajuela. La UTN-SA dispone de una finca de 540 ha, de las cuales 200 ha son zonas de protección o plantaciones forestales, con una altitud que varía desde los 250 a 650 msnm. La UTN-SA es una sede universitaria que se especializa en impartir carreras agropecuarias y agroindustriales, además cuenta con 110 instalaciones, entre ellas dos plantas agroindustriales, laboratorios, oficinas, aulas, residencias para más de 300 estudiantes, 1000 semovientes (ganado de carne, ganado lechero, búfalos, caballos, cerdos, cabras y ovejas), 789 estudiantes y 200 funcionarios en el 2015.

Fuentes de emisión. Se identificó ocho fuentes de emisiones de gases con efecto invernadero (semovientes, combustibles, gas licuado de petróleo (GLP), fertilizantes nitrogenados, extintores de $\mathrm{CO}_{2}$, aguas residuales, electricidad y residuos ordinarios, los cuales se dividen en directas e indirectas. Se determinó la cantidad de estudiantes promedio, utilizando los reportes del departamento de registro universitario del 2012 al 2015. La metodología aplicada es la recomendada por el IPCC (2006) y se utilizaron los factores de emisión según IMN (2016).

Emisiones directas. Se estimó las emisiones de seis fuentes directas: semovientes (ganado de carne, ganado de leche, caballos, búfalos, cabras, ovejas y cerdos), combustibles fósiles (diésel y gasolina), gas licuado de petróleo (GLP), fertilizantes nitrogenados, extintores de $\mathrm{CO}_{2} \mathrm{y}$ aguas residuales.

Emisiones generadas por semovientes. Se estimaron las emisiones generadas por cuatro especies de rumiantes: bovinos, caprinos, ovinos, bufalinos y dos monogástricos: cerdos y equinos. Los datos de semovientes se obtuvieron de los registros del departamento de control de bienes de la UTN-SA, tomando como punto de referencia el inventario anual que se realiza en el mes de octubre, con el cual se determinó las categorías de cabezas por especie animal. La metodología aplicada es la recomendada por el IPCC (2006) y se utilizaron los factores de emisión según IMN (2016). En el caso de los semovientes se estima la fermentación entérica y manejo del estiércol (El termino estiércol incluye orina + excremento). El cálculo de emisiones de $\mathrm{CO}_{2}$ e debido a este aspecto se realizó mediante la siguiente fórmula:

$$
\begin{gathered}
\text { Ton } \mathrm{CO}_{2} \mathrm{e}=[\mathrm{Cb} * \mathrm{FG} \text { proc dig })+ \\
(\mathrm{Cb} * \mathrm{FG} \text { man est })] * 21 / 1000 \mathrm{~kg} / \text { ton }
\end{gathered}
$$


Dónde:

Ton $\mathrm{CO}_{2} \mathrm{e}:$ Toneladas de dióxido de carbono equivalente.

Cb: Cabezas animal.

FGprocdig: Factor de emisión oficial del IMN, kilogramos de $\mathrm{CH} 4$ emitido por el ganado en el proceso digestivo por cabeza animal.

$\mathrm{Cb}$ : Cabezas animal.

FG manest: Factor de emisión oficial del IMN, kilogramos de $\mathrm{CH} 4$ emitido por cabeza animal en el manejo de estiércol.

21: Potencial de calentamiento global del $\mathrm{CH} 4$ para conversión a $\mathrm{CO}_{2}$.

1000 kg/ton: Factor de kilogramos a toneladas.

Emisiones generadas por combustible. Se cuantificó el consumo en litros de combustible/ año (gasolina y diésel), donde la gasolina es empleada principalmente en motores pequeños y el diésel en la mayoría de los vehículos y caldera. Para el cálculo de emisiones se utilizó la metodología sugerida por el IPCC (2006) y factores de emisión del IMN (2016). Los datos de consumo en litros de combustibles fueron obtenidos de los registros de la unidad de transportes de la UTN-SA. El cálculo de emisiones de $\mathrm{CO}_{2}$ e por el consumo de diésel y gasolina se realizó con la siguiente fórmula:

$$
\text { Ton } \mathrm{CO}_{2} \mathrm{e}=\left(\mathrm{L}^{*} \mathrm{FCO}^{2}\right) / 1000 \mathrm{Kg} / \mathrm{ton}
$$

Dónde:

Ton $\mathrm{CO}_{2} \mathrm{e}$ : Toneladas de dióxido de carbono equivalente.

L: Litros de combustible diésel o gasolina $\mathrm{FCO}_{2}$ : Factor de emisión oficial del IMN, kilogramos de $\mathrm{CO}_{2}$ emitido/cada litro de diésel o gasolina.

$1000 \mathrm{Kg} /$ ton: Factor de kilogramos a toneladas

Emisiones generadas por gas licuado de petróleo (GLP). Para el cálculo de emisiones se utilizó la metodología sugerida por el IPCC (2006) y factores de emisión del IMN (2016). El consumo en litros de GLP obtuvo mediante la sumatoria de todas las facturas aportadas por el concesionario del comedor institucional de la
UTN-SA. El cálculo de emisiones de $\mathrm{CO}_{2}$ e se realizó con la siguiente fórmula:

$$
\text { Ton } \mathrm{CO}_{2} \mathrm{e}=\left(\mathrm{LGLP}^{*} \mathrm{FGLP}\right) / 1000 \mathrm{Kg} / \mathrm{ton}
$$

Dónde:

Ton $\mathrm{CO}_{2} \mathrm{e}$ : Toneladas de dióxido de carbono equivalente.

LGLP: Litros de Gas Licuado de Petróleo consumidos.

FGLP: Factor de emisión oficial del IMN, kilogramos de $\mathrm{CO}_{2}$ emitido por cada litro GLP

$1000 \mathrm{Kg} /$ ton: Factor de kilogramos a toneladas.

Emisiones generadas por la aplicación de fertilizantes nitrogenados. Se cuantificó los kilogramos de fertilizantes nitrogenados utilizados que favorecen la emisión del $\mathrm{N}_{2} \mathrm{O}$. Esto se obtuvo del registro del almacén de proveeduría de la UTN-SA. Para la determinación se utilizó la metodología recomendada por el IPCC (2006) y el $1 \%$ de nitrógeno volatilizable por concepto de otros pastos (IMN,2016). El cálculo de emisiones de $\mathrm{CO}_{2}$ e debido a la utilización de fertilizantes se realizó mediante la siguiente fórmula:

$$
\begin{aligned}
& \text { Ton } \mathrm{CO}_{2} \mathrm{e}=\mathrm{NSF} * 45 \mathrm{Kg} * \% \mathrm{~N} / 100 * \\
& 1 \% / 100 * 1,5714 * 310 / 1000 \mathrm{Kg} / \text { ton }
\end{aligned}
$$

Dónde:

Ton $\mathrm{CO}_{2} \mathrm{e}$ : Toneladas de dióxido de carbono equivalente.

NSF: Cantidad de sacos de fertilizante.

$45 \mathrm{Kg}$ : Peso en kilogramos de un saco de fertilizante.

$\% \mathrm{~N}$ : Porcentaje de nitrógeno presente en el fertilizante.

1\%: Porcentaje de nitrógeno que se volatiliza.

1,5714: Transformación del nitrógeno N2 a óxido nitroso N2O.

310: Transformación de óxido nitroso N2O a dióxido de carbono $\mathrm{CO}_{2}$

$1000 \mathrm{Kg} /$ ton: Factor de kilogramos a toneladas.

Emisiones generadas por recargas de extintores de $\mathrm{CO}_{2}$. Se determinó el número de extintores y su capacidad, según registros del área de seguridad de la UTN-SA. Los extintores de 
$\mathrm{CO}_{2}$ son recargados una vez al año. Para la determinación se utilizó la metodología recomendada por el IPPC (2006) y los índices del IMN (2016). El cálculo de emisiones de $\mathrm{CO}_{2}$ e se realizó con la siguiente fórmula:

$$
\text { Ton } \mathrm{CO}_{2} \mathrm{e}=\left(\mathrm{N} 1 * \mathrm{CP}^{*} \mathrm{RA} * \mathrm{~F}\right) / 1000 \mathrm{Kg} / \mathrm{ton}
$$

Dónde:

Ton $\mathrm{CO}_{2}$ e: Toneladas de dióxido de carbono equivalente.

N1: Cantidad de extintores.

CP: Capacidad promedio en kilogramos por extintor

RA: Recargas al año

F: Factor de emisión oficial del IMN, kilogramos de $\mathrm{CO}_{2}$ emitido.

$1000 \mathrm{Kg} /$ ton: Factor de kilogramos a toneladas.

Emisiones por tratamiento de aguas residuales. La UTN-SA cuenta con dos lagunas de oxidación para tratar las aguas residuales. La información fue obtenida de los reportes operacionales aportados por la unidad de gestión ambiental de la UTN-SA. Para la determinación se utilizó la metodología recomendada por el IPCC (2006) y los índices del IMN (2016). El cálculo de emisiones de $\mathrm{CO}_{2}$ e se realizó mediante la siguiente fórmula:

$$
\text { Ton } \mathrm{CO}_{2} \mathrm{e}=\mathrm{Np} * \mathrm{FAr} * 21 / 1000 \mathrm{Kg} / \text { ton }
$$

Dónde:

Ton $\mathrm{CO}_{2} \mathrm{e}$ : Toneladas de dióxido de carbono equivalente.

Np: Número de estudiantes promedio anual.

FAr: Factor de emisión oficial del IMN, kilogramos de $\mathrm{CH} 4$ emitido por cada persona al año.

21: Potencial de calentamiento global del $\mathrm{CH} 4$ para conversión a $\mathrm{CO}_{2}$.

$1000 \mathrm{Kg} /$ ton: Factor de kilogramos a toneladas.

Emisiones indirectas. Se determinó dos fuentes de emisiones indirectas: el consumo de electricidad y los residuos ordinarios.

Emisiones por consumo eléctrico. El consumo eléctrico en KWh se estimó por medio de la sumatoria de todos los recibos emitidos por el Instituto Costarricense de Electricidad correspondiente a los años del 2012 al 2015 de la UTN-SA. Para la determinación se utilizó la metodología recomendada por el IPCC (2006) y los índices del IMN (2016). El cálculo de emisiones de $\mathrm{CO}_{2}$ e se realizó con la siguiente fórmula:

$$
\text { Ton } \mathrm{CO}_{2} \mathrm{e}=\mathrm{kWh} * \mathrm{FKWh} / 1000 \mathrm{Kg} / \text { ton }
$$

Dónde:

Ton $\mathrm{CO}_{2} \mathrm{e}$ : Toneladas de dióxido de carbono equivalente.

kWh: Kilowatts hora de energía eléctrica consumida.

FKWh: Factor de emisión oficial del IMN, kilogramos de $\mathrm{CO}_{2}$ emitido/ cada kilowatt hora consumido.

$1000 \mathrm{Kg} /$ ton: Factor de kilogramos a toneladas.

Emisiones por residuos ordinarios. Los residuos ordinarios o residuos domésticos generan emisión indirecta ya que son enviados a un relleno sanitario, para su determinación se emplearon los reportes de la empresa WPP Continental de Costa Rica y de la municipalidad de Atenas que son los encargados de recolectar y llevar los residuos de la UTN-SA a un relleno sanitario. Para la determinación se utilizó la metodología recomendada por el IPCC (2006) y los índices del IMN (2016). El cálculo de emisiones de $\mathrm{CO}_{2}$ e mediante este aspecto se realizó con la siguiente fórmula:

$$
\text { Ton } \mathrm{CO}_{2} \mathrm{e}=\mathrm{NRs} * \mathrm{FRs} * 21 / 1000 \mathrm{Kg} / \text { ton }
$$

Dónde:

Ton $\mathrm{CO}_{2} \mathrm{e}$ : Toneladas de dióxido de carbono equivalente.

NRs: Cantidad de residuos sólidos en kilogramos enviados al relleno sanitario.

FRs: Factor de emisión oficial del IMN, kilogramos de $\mathrm{CH} 4$ emitido/kilogramo de residuos sólidos.

21: Potencial de calentamiento global del $\mathrm{CH} 4$ para conversión a $\mathrm{CO}_{2}$.

$1000 \mathrm{Kg} /$ ton: Factor de kilogramos a toneladas. 
Relación de $\mathrm{tCO}_{2}$ emitidas con respecto a la cantidad de estudiantes matriculados del 2012 al 2015. La cantidad de estudiantes matriculados promedio por año se obtuvieron del departamento de registro de la UTN-SA. El cálculo de emisiones de $\mathrm{CO}_{2}$ e promedio por estudiante por año se realizó con la siguiente fórmula:

$$
\mathrm{tCO}_{2} \mathrm{e} \text { por estudiante }=\mathrm{tCO}_{2} \mathrm{e} / \mathrm{NEs}
$$

Dónde:

$\mathrm{tCO}_{2}$ e: Toneladas de dióxido de carbono equivalente emitidas por año.

NEs: Cantidad promedio de estudiantes matriculados por año.

Emisiones de $\mathrm{tCO}_{2}$ e por estudiante de universidades latinoamericanas como punto de referencia. Se escogió seis universidades latinoamericanas, de las cuales cuatro son de Costa Rica, una de Chile y una de Colombia, todas aplicaron los protocolos IPCC 2006 y tiene relación con el sector agropecuario. Para el análisis de la información se utilizó reportes publicados por las universidades que indican las emisiones por estudiante o se obtuvo la información de forma indirecta. Para estimar las emisiones de forma indirecta se dividieron las emisiones totales entre los estudiantes matriculados.

Análisis estadístico. Para analizar comparativamente los valores de las emisiones de $\mathrm{tCO}_{2} \mathrm{e}$ y su relación con las variables número de estudiantes y año de emisión, se utilizaron modelos de regresión lineal, con el programa estadístico $\mathrm{R}$ versión 3.4.3.

\section{Resultados}

El 93\% de las emisiones de la UTN-SA son generadas por tres fuentes: semovientes con $57 \%$, residuos ordinarios con $19 \%$ y combustibles con $16 \%$, el restante de las fuentes emite el $7 \%$ (cuadro 1 ).

En general las emisiones totales aumentaron con el número de estudiantes, pero al desglosarlas se determinan tres patrones: las fuentes de emisión de GEI que aumentan las emisiones

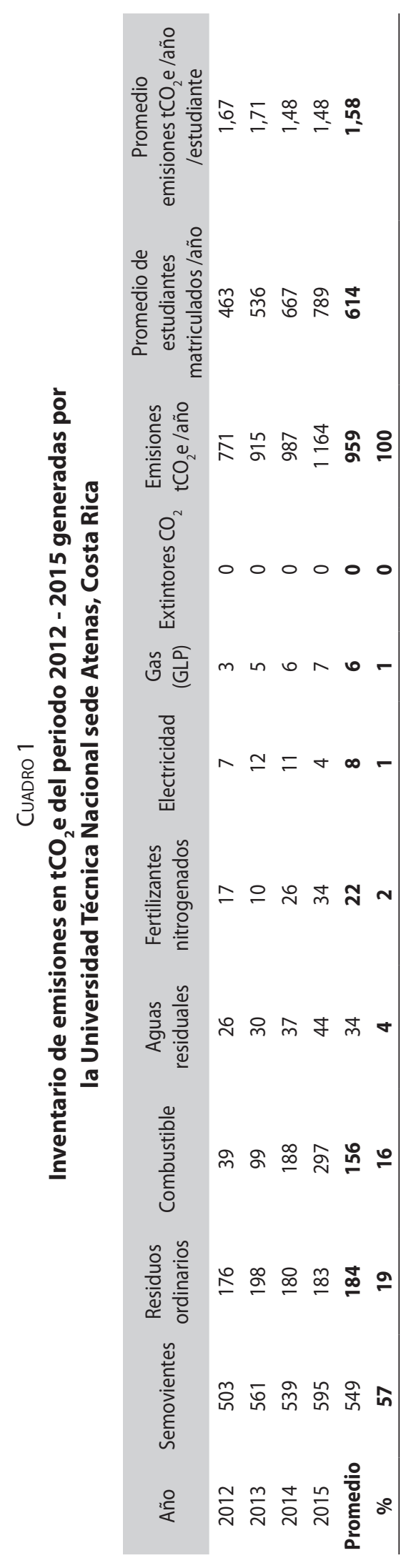




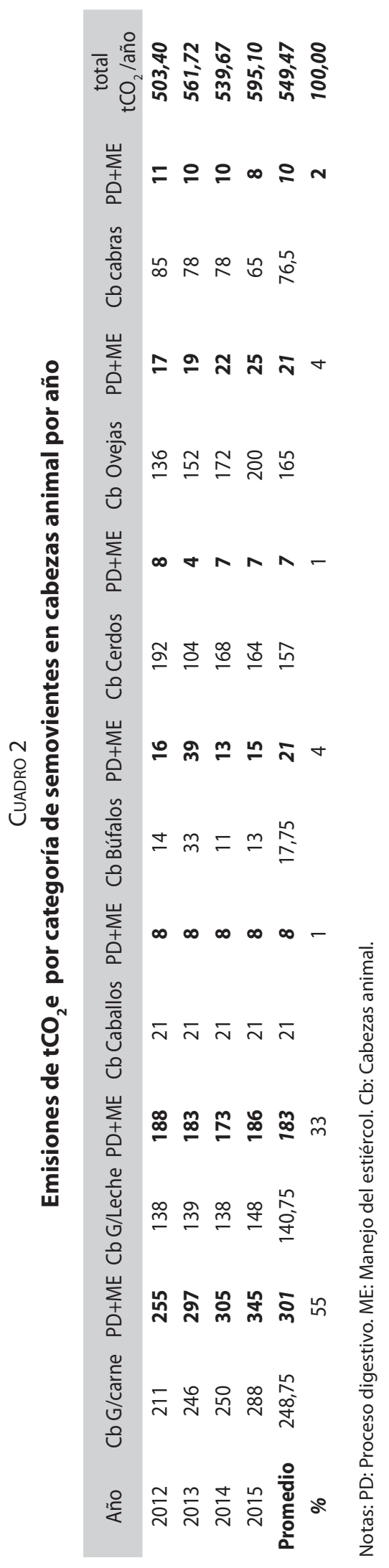

son los semovientes, residuos ordinarios, combustibles, aguas residuales, fertilizantes y el gas (GLP). Las que se mantienen son los extintores. La fuente de emisión que disminuye es la electricidad debido a una disminución en el factor térmico (Cuadro 1). Los semovientes son el principal emisor, debido a la cantidad de animales y factor elevado de conversión de metano a $\mathrm{CO}_{2}$, generando en promedio $549 \mathrm{tCO}_{2} \mathrm{e}$ al año, equivalente al $57 \%$ de las emisiones totales (Cuadro1). Dentro de los semovientes, el hato de ganado de carne ocupa el primer lugar de emisiones con $55 \%$, seguido por el hato de ganado de leche con $33 \%$, el resto de los semovientes generan el $12 \%$ (Cuadro 2)

Los residuos ordinarios ocupan el segundo lugar en importancia con un $19 \%$ del total de las emisiones, siendo principalmente generados por los estudiantes, funcionarios, visitantes, actividades académico-productivas. A lo largo del estudio, presenta un comportamiento muy estable a pesar del crecimiento de estudiantes (Cuadro 1).

Los combustibles ocupan el tercer puesto en importancia con un 16\% del total de emisiones, donde el consumo se distribuye $73 \%$ diésel y el $27 \%$ gasolina. Además, presenta el mayor crecimiento porcentual comparado con las demás fuentes emisoras. El diésel es consumido principalmente por vehículos, maquinaria y equipo agrícola y la caldera de la planta de lácteos; la gasolina es utilizada en motores de dos tiempos como chapedadoras y motosierras (Cuadro 1). En la Figura. 1, la relación del número de estudiantes y las emisiones es positiva con respecto al consumo de combustible y se interpreta cuantitativamente que por cada estudiante que se agrega a la población de la UTN-SA incrementan las emisiones por el uso de combustibles en $0,77 \mathrm{tCO}_{2}$.

En la Figura 2, la relación del número de estudiantes y las emisiones totales es positiva, siendo que por cada estudiante que se agrega a la población, se incrementa en $1,11 \mathrm{tCO}_{2}$ anuales de emisiones.

En la Figura 3 se presenta una tendencia a disminuir y estabilizar las emisiones por estudiante a partir del 2014 en 1,48 $\mathrm{tCO}_{2} \mathrm{e}$ con 


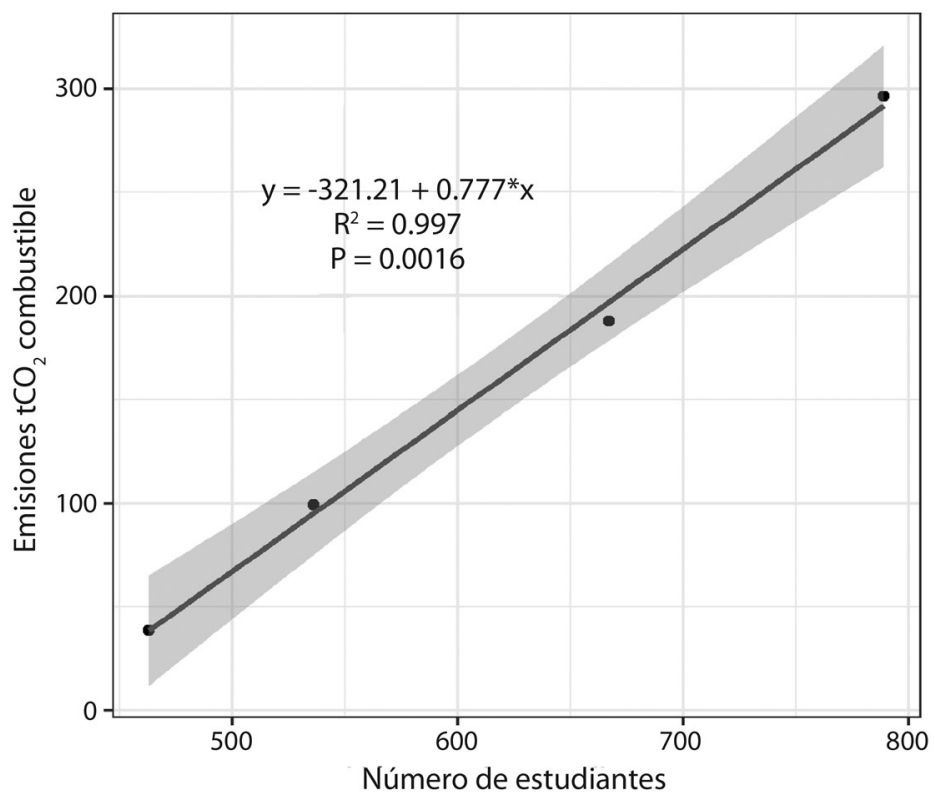

Figura 1. Emisiones en $\mathrm{tCO}_{2}$ e anuales generadas por combustibles y relación con el número de estudiantes matriculados del 2012 al 2015 en la UTN-SA.

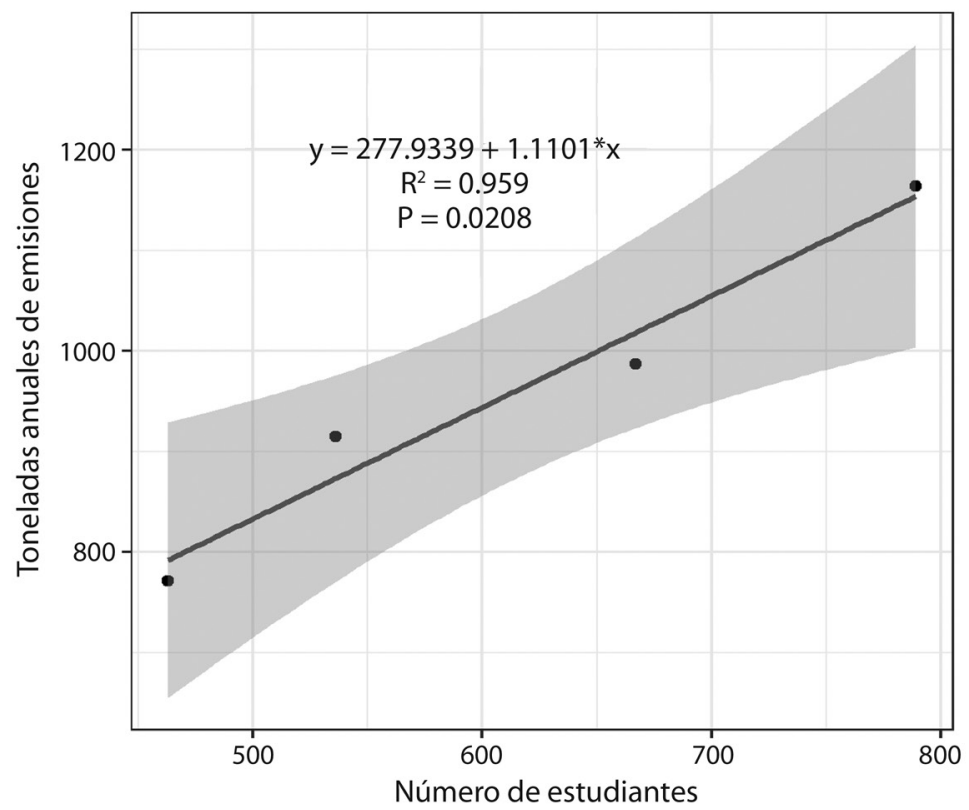

Figura 2. Emisiones totales en $\mathrm{tCO}_{2}$ e anuales y su relación con el número de estudiantes matriculados del 2012 al 2015 en la UTN-SA.

respecto al número de estudiantes matriculados del 2012 al 2015 UTN-SA, lo anterior debido a que porcentualmente ingresan más estudiantes y las fuentes de emisiones no incrementan al mismo ritmo, es decir al ingresar más estudiantes las fuentes de emisiones no incrementan al mismo tiempo.

En el 2013 los niveles de emisión por estudiante son los más altos con respecto a los demás años, debido a que el ingreso de estudiantes fue 


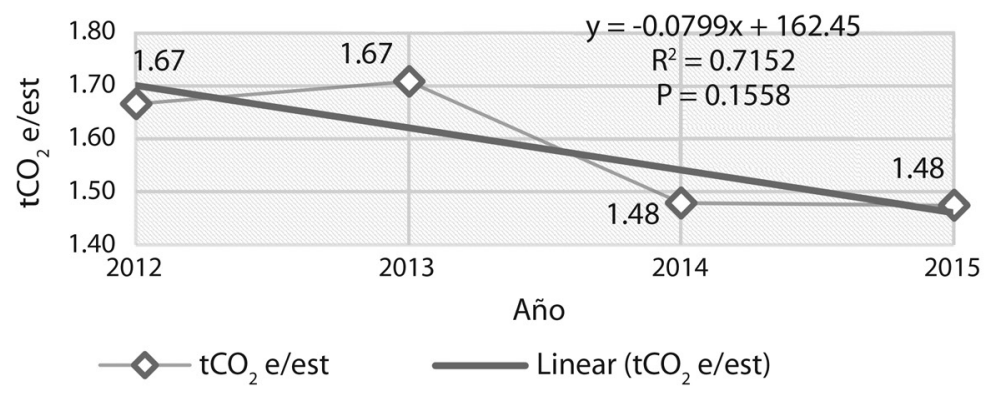

Figura 3. Promedio anual de emisiones por estudiante.

el más bajo de los cuatro años y las emisiones de semovientes, desechos ordinarios y combustible aumentaron a causa de un mayor número de semovientes del hato de ganado de carne, incremento en la capacidad de residencias y por ende mayor generación de desechos ordinarios y un mayor número de actividades académicas que requerían de combustible para realizarlas.

En el cuadro 3, al referenciar las emisiones en $\mathrm{tCO}_{2}$ e/estudiante de seis universidades latinoamericanas, cuatro de ellas de Costa Rica y dos Sudamericanas, se aprecia claras diferencias que hacen difícil su comparación, con emisiones que van desde 0,11 hasta 4,6 $\mathrm{tCO}_{2}$ e/estudiante/ año, la UTN-SA ocupa el segundo lugar en importancia con $1,48 \mathrm{tCO}_{2}$ e y la EARTH el primero con 4,6 $\mathrm{tCO}_{2} \mathrm{e}$, siendo ambas universidades similares por impartir educación agropecuarioagroindustrial. Al referenciar las emisiones con las otras cinco universidades, las diferencias son significativas, ya que estas cuentan con miles de estudiantes, son carreras principalmente administrativas, que les permiten una menor emisión por estudiante. En nuestro caso la cantidad de estudiantes es menor y las carreras son principalmente agropecuarias e industriales, las cuales

\section{CUADRO 3}

\section{Emisiones promedio en $\mathrm{tCO}_{2}$ e por estudiante de seis universidades latinoamericanas y su comparación con la Universidad Técnica Nacional sede Atenas}

\begin{tabular}{|c|c|c|c|c|c|}
\hline Universidad & $\begin{array}{c}\text { Año de } \\
\text { comparación }\end{array}$ & $\begin{array}{l}\text { Número de } \\
\text { estudiantes }\end{array}$ & $\begin{array}{l}\text { Total de } \mathrm{tCO}_{2} \mathrm{e} \\
\text { emitidas /año }\end{array}$ & $\begin{array}{l}\text { Promedio ajustado } \\
\text { en } \mathrm{tCO}_{2} \mathrm{e} / \text { Estudiante }\end{array}$ & Referencia bibliográfica \\
\hline Universidad de Costa Rica & 2013 & 39730 & 4538 & 0,11 & $\begin{array}{l}\text { Ruíz. C. (2013), Estado } \\
\text { de la Nación (2015) }\end{array}$ \\
\hline $\begin{array}{l}\text { Universidad Nacional } \\
\text { de Costa Rica }\end{array}$ & 2014 & 17879 & 3568 & 0,20 & $\begin{array}{l}\text { Estado de la Nación (2015); } \\
\text { Chavarría F, Molina O, } \\
\text { Gamboa, R. Rodríguez, J. } \\
\text { 2016. (2016) }\end{array}$ \\
\hline $\begin{array}{l}\text { Universidad Politécnica de } \\
\text { Cartagena en Colombia }\end{array}$ & 2014 & 7458 & 2825 & 0,38 & $\begin{array}{l}\text { Universidad Politécnica } \\
\text { de Cartagena (2014). }\end{array}$ \\
\hline $\begin{array}{l}\text { Instituto Tecnológico } \\
\text { de Costa Rica }\end{array}$ & 2013 & 10043 & 3860 & 0,38 & $\begin{array}{l}\text { Ruíz. C. (2013) y Estado } \\
\text { de la Nación (2015) }\end{array}$ \\
\hline $\begin{array}{l}\text { Universidad Tecnológica } \\
\text { Metropolita de Chile }\end{array}$ & 2010 & 5954 & 7204 & 1,21 & $\begin{array}{l}\text { Ortúzar. P, Toledo. C y } \\
\text { Yurisch T(2010) }\end{array}$ \\
\hline $\begin{array}{l}\text { Escuela Agricultura de la } \\
\text { Región Tropical Húmeda. } \\
\text { Costa Rica }\end{array}$ & 2015 & 432 & 1990 & 4,60 & $\begin{array}{l}\text { EARTH (2018) y EARTH, } \\
\text { Memoria anual (2015) }\end{array}$ \\
\hline $\begin{array}{l}\text { Universidad Técnica Nacional } \\
\text { sede Atenas. Costa Rica }\end{array}$ & 2014 & 667 & 987 & 1,48 & \\
\hline
\end{tabular}

Fuente: elaboración propia. 
no permiten grupos grandes de práctica (8 a 15), requieren insumos agrícolas y combustibles, de semovientes y manejo de fincas que incrementan las emisiones por estudiante. Por lo tanto, bajo las condiciones actuales no es posible realizar una comparación objetiva entre universidades.

\section{Discusión}

Al ser la UTN-SA una sede universitaria con carreras mayoritariamente agropecuarias, es de esperar que los semovientes sean la principal fuente de emisión, caso contrario ocurre con otras universidades como por ejemplo la universidad de Costa Rica, sede occidente, con carreras principalmente administrativas, donde su mayor emisor es la electricidad, segundo los combustibles y no presentan emisiones por semovientes (Chassoulla et al., 2017), en cambio, la Universidad Nacional (UNA) reporta como su principal emisor los viajes aéreos, segundo combustible, tercera electricidad y los semovientes ocupan el octavo lugar con un aporte del $0,5 \%$ del total de sus emisiones (Chavarría, et al, 2016).

Aunque los residuos ordinarios ocupan el segundo lugar de importancia es un resultado transitorio, entre la salida de funcionarios y el incremento del número estudiantes, con una tendencia a disminuir en el corto plazo. En cuanto al porcentaje y tipo de combustible la UTN-SA coincide con lo reportado por Chassoulla et al. (2017), donde la Universidad de Costa Rica sede Occidente (UCR-SO) presenta porcentajes de consumos idénticos, siendo $73 \%$ diésel y $23 \%$ gasolina, pero los porcentajes de emisiones globales varía por combustible entre universidades, la UCR-SO reporta un 29\%, UNA 23\% y la UTN-SA el 16\% (Chavarría, et al., 2016).

Durante el 2014 y 2015, el promedio de emisión por estudiante en la UTN-SA se estabilizó en $1,48 \mathrm{tCO}_{2}$ e/estudiante, a pesar de que la población estudiantil incrementó un 18,3\%, siendo similar a lo reportado por Chavarría, et al. (2016) de la UNA, donde del 2013 al 2014 la población estudiantil creció un $15,41 \%$ y sus emisiones igualmente presentaron una tendencia a estabilizar a pesar del crecimiento de estudiantes, al parecer es una tendencia normal. Cabe destacar que, al referenciar y ajustar la huella de carbono por estudiante de seis universidades latinoamericanas con respecto a la UTN-SA se determinó que no es posible estandarizar los datos para realizar una comparación real con respecto a sus emisiones, debido a las diferentes condiciones y características propias de cada universidad, por lo tanto, solo se utilizó como punto de referencia. Al final del estudio se determinó que a mayor número de estudiantes las emisiones totales aumentan, pero disminuyen las emisiones promedio por estudiante, por lo tanto, se acepta la hipótesis planteada.

\section{Conclusiones y Recomendaciones}

De acuerdo con el análisis de los datos se puede concluir que el promedio anual de emisiones del 2012 al 2015 de la UTN-SA es de $959 \mathrm{tCO}_{2} \mathrm{e}$, con una máxima de $1164 \mathrm{tCO}_{2} \mathrm{e}$ en el 2015 y con tendencia a incrementar y el $92 \%$ de las emisiones de $\mathrm{tCO}_{2}$ e en la UTN-SA se concentran en tres fuentes emisoras, los semovientes $(57 \%)$, residuos ordinarios $(19 \%)$ y los combustibles $(16 \%)$.

Las fuentes de emisión que incrementan al aumentar el número de estudiantes según su orden de importancia son: combustibles, GLP, fertilizantes nitrogenados, aguas residuales, semoviente y residuos ordinarios. La fuente de emisión que no presenta variación son los extintores y la que disminuye al aumentar el número de estudiantes es la electricidad por disminución en el factor térmico.

Por otro lado, la cantidad de $\mathrm{tCO}_{2}$ e promedio por estudiante disminuye al aumentar la población estudiantil a partir del 2014, con tendencia a estabilizarse en 1,48 $\mathrm{tCO}_{2} \mathrm{e}$. Siendo el promedio de emisiones en $\mathrm{tCO}_{2} \mathrm{e}$ de los cuatro años del estudio por estudiante en la UTN-SA es de 1,58 $\mathrm{tCO}_{2} \mathrm{e}$, siendo inferior al 2,41 $\mathrm{tCO}_{2} \mathrm{e}$ promedio per cápita nacional.

Se recomienda ahondar en la determinación de la capacidad de fijación de $\mathrm{tCO}_{2}$ e de la UTN-SA para realizar el balance de Gases de efecto invernadero y el establecimiento de un 
programa de mitigación de los GEI en la UTNSA en cuanto a:

- Manejo de residuos ordinarios y aguas residuales, como por ejemplo mejorar el programa para el manejo integral de los residuos sólidos y analizar la factibilidad de construir una planta de tratamiento para las aguas residuales.

- Combustibles y gas GLP, capacitar al personal en el manejo eficiente vehicular $\mathrm{y}$ planificar y unificar giras que permitan reducir el consumo de combustible. Así como sistematizar la renovación de las unidades automotoras y otros equipos, por unidades o equipos más eficientes. También fomentar el uso de equipos de transporte no contaminantes como por ejemplo bicicletas o vehículos eléctricos.

- Energía eléctrica, establecer un programa de reducción y uso eficiente de la energía eléctrica e implementar proyectos de energías eficientes o alternativas, tales como paneles solares.

- Con los extintores, es necesario capacitar a funcionarios y estudiantes en el uso responsable de extintores.

\section{Referencias}

Banco Mundial. (2015). Emisiones de todos los gases de efecto invernadero (toneladas métricas per cápita). Indicadores del desarrollo mundial. Centro de Análisis de Información sobre Dióxido de Carbono, División de Ciencias Ambientales del Laboratorio Nacional de Oak Ridge, Tennessee, Estados Unidos. Recuperado de: http://datos.bancomundial.org/indicador/EN.ATM.CO2E.PC/countries/

Chavarría F, Molina O, Gamboa, R. \& Rodríguez, J. (2016). Medición de la huella de carbono de la Universidad Nacional de Costa Rica para el periodo 2012-2014. Rumbo a la carbono neutralidad. Heredia, Costa Rica: Editorial UNICIENCIA. Universidad Nacional. Recuperado de:http://www.revistas.una.ac.cr/index.php/uniciencia/article/view/7756/9169\#BancoMundial2015-Emisiones-de-todos
Chassoul M., Marín R., Morales M. \& Fallas, F. (2017). Cuantificación de gases de efecto invernadero en la Sede de Occidente de la Universidad de Costa Rica. (15), 1, 69-77. Costa Rica. Recuperado de: http://Downloads/DialnetCuantificacionDeGases DeEfectoInvernaderoEnLaSedeDe-6039012.pdf

Cuervo,B.(2013)Elimparableaumentodelapoblaciónmundial. Recuperado de: http://www.otromundoesposible. net/el-imparable-aumento-de-la-poblacion-mundial/

Cuervo, B. (2015). La población mundial y sus proyecciones demográficas. Recuperado de: http://www. otromundoesposible.net/el-imparable-aumentode-la-poblacion-mundial/

Dobles, R. (2008). Estrategia nacional de cambio climático. San José, Costa Rica: Ministerio del Ambiente y Energía.

Escuela de Agricultura de la Región Tropical Húmeda. (2008). Manual Neutralidad del Carbono. LimónCosta Rica.

Escuela de Agricultura de la Región Tropical Húmeda. (2015). Memoria anual. Edición 25 aniversario. Limón-Costa Rica. Recuperado de: https://www. earth.ac.cr/es/about-earth/earth-facts/memoria-anual/ report-aniversary-edition/

Escuela de Agricultura de la Región Tropical Húmeda. (2018). Informe PBAE Categoría Comunidad Clima Neutral . Limón, Costa Rica.

Estado de la Nación (2015). Capítulo 4: Evolución de la Educación superior. Costa Rica. Recuperado de: http://www.estadonacion.or.cr/educacion2015/assets/ cap-4-ee-2015.pdf

Fresneda, C. (2017). El2016 fija un nuevo récord de la temperatura global. El Diario. Recuperado de.Mundohttp:// www.elmundo.es/ciencia/2017/01/18/587faed4461 63fa1518b45c9.html. Londres Inglaterra.

Instituto Meteorológico Nacional. (2016). Factores de gases de efecto invernadero en Costa Rica. Recuperado de http://www.imn.global.ogr.com.

IPCC (2006). Directrices del IPCC de 2006 para los inventarios nacionales de gases de efecto invernadero. Recuperado de:http://www.ipcc-nggip.iges.or.jp/ public/2006gl/spanish/index.html 
IPCC (2007). Cambio Climático 2007. Informe de síntesis. Recuperado de: https://www.ipcc.ch/pdf/assessmentreport/ar4/syr/ar4_syr_full_report.pdf

Ruíz. C. (2013). UCR y TC, aspiran a ser carbono neutral. Recuperado de: http://www.nacion.com/vivir/ambiente/UCR-apuesta-carbono neutral_0_1375262666.html

R Development Core Team (2008). R: A language and environment for statistical computing. Vienna, Austria: R Foundation for Statistical Computing. Recuperado de: URL http://www.R-project.org.

Ministerio del Ambiente y Energía. (2016). Hacia la Carbono Neutralidad 2021. Dirección de Cambio Climático. Recuperado de http://cambioclimaticocr. com

Kioto (1997) Protocolo de Kyoto de la convención Marco de las naciones unidas sobre el Cambio climático.
Recuperado de: https://unfccc.int/resource/docs/convkp/kpspan.pdf

Universidad Nacional de Heredia. (2011). Reportes de emisiones de gases efecto invernadero. San José, Costa Rica: UNA.

Ortúzar. P, Toledo. C. \& Yurisch T, 2010. Estudiantes miden emisión de huella de carbono en la UTEM. Universidad Tecnológica Metropolitana de Chile. Recuperado de: https://aldia.utem.cl/estudiantes-miden-emision-de-huella-de-carbono-en-la-utem/

Universidad Politécnica de Cartagena. (2014). Huella de carbono de la Universidad Politécnica de Cartagena. Vicerrectoría de investigación. Colombia. Recuperado de:http://www.upct.es/gestionserv/inter /web_servinfraestructuras_fich/doc_secciones /268huella-de-carbono-u 